\title{
Relationships between the Parity and Pelvimetry of Egyptian Buffalo Cows: Prediction of Dystocia and Estimation of Age
}

\author{
Ramadan G. Sary ${ }^{1}$, Hisham A. Abdelrahman², Ragab H. Mohamed ${ }^{3}$, Ahmed M. Hussien ${ }^{4}$, \\ Hassan A. Hussein ${ }^{5}$ and Karim M. Khalii ${ }^{1,6,{ }^{*}}$ \\ ${ }^{1}$ Anatomy and Embryology Department, Faculty of Veterinary Medicine, Cairo University, Giza 12211, Egypt \\ ${ }^{2}$ Department of Veterinary Hygiene and Management, Faculty of Veterinary Medicine, Cairo University, Giza \\ 12211, Egypt \\ ${ }^{3}$ Theriogenology Department, Faculty of Veterinary Medicine, Aswan University, Aswan, Egypt \\ ${ }^{4}$ Department of Toxicology and Forensic Medicine, Faculty of Veterinary Medicine, Cairo University, Giza \\ 12211, Egypt \\ ${ }^{5}$ Theriogenology Department, Faculty of Veterinary Medicine, Assiut University, Assiut, Egypt \\ ${ }^{6}$ Department of Veterinary Medicine, College of Applied and Health Sciences, A'Sharqiyah University, Ibra, \\ Sultanate of Oman
}

\begin{abstract}
Background: The current study aimed to determine the most strongly correlated variable of pelvimetry with the parity in our native breed Egyptian buffaloes.

Methods: The study was conducted on 36 female buffaloes (nullipara, $n=14$, primipara $n=6$ and pluripara, $n=16$ with $2-4$ births) aged between $<15$ months, $n=15$ and 65 months, $n=21$. The internal and external pelvic measurements were obtained using the rice pelvimeter and Freeman's measuring tape.

Results: Strong positive linear relationships were found for the distance between ischiatic tuberosities and the distance between sacral tubercles with the correlation coefficients of 0.64 and 0.62 , respectively. The conjugate diameter increased progressively with the age and number of births, with a correlation coefficient of 0.96 . The pelvic area had a very strong positive linear relationship with a correlation coefficient of 0.89 . The linear combination of the predictor variable (conjugate diameter), to predict the number of birth was developed successfully.

Conclusion: The strong relationship between the conjugate diameter and the number of births could be employed to predict the dystocia and estimate the age of female buffalo. Furthermore, these findings could be aid paleontologists in studying buffalo fossils.
\end{abstract}

Keywords: Bubalus bubalis, Pelvis, Dystocia, Forensic Anatomy, Applied anatomy.

\section{INTRODUCTION}

Buffalo (Bubalus bubalis) plays a prominent role in rural livestock production in Egypt. In buffaloes, the incidence of dystocia, difficult parturition is higher than in cows [1]. Calving ease may be influenced by the level of development of the pelvic area and reproductive organs, especially in heifers [2]. Studies were shown that the internal pelvic area is one of the best predictions of dystocia in heifers and the best method of dealing with calving problems is to avoid them [3]. Pelvimetry is one of the most important tools to consider in a breeding program. Pelvimetry means the measurement of the dimensions and capacity of the pelvis, either externally, internally, or both [4]. The pelvic sizes of cows vary according to their reproductive stage $[5,6]$. Close correlations between calving ease and pelvic measurements and its shape

*Address correspondence to this author at the College of Applied and Health Sciences, A'Sharqiyah University, P.O. Box 42, Postal Code: 400 Ibra, Sultanate of Oman; Tel: +968 97790855; E-mail: Karim.Khalil@asu.edu.om, Karim.Khalil@vet.cu.edu.eg were observed [7]. Dams with larger pelvic areas practice less calving difficulty, and a narrow pelvis can disturb the delivery, making it difficult to expel the fetus $[8,9]$.

The pelvis is a multiple function osteoligamentous complexes [10]. In ruminants, the pelvis is classified as dolichopelvic because the sacropubic (conjugate) diameter in females is larger than the median bi-iliac (transverse) breadth [11]. The bony structure of the pelvis consists of the sacrum and first three coccygeal vertebrae dorsally and the coxal bones (left and right) formed by the fusion of ilium laterally ischium, and pubis ventrally [11]. The articulation between coxal bones (right and left) is an amphiarthrosis called pelvic symphysis [11]. Pelvimetry represents an applicable versatile indicator to reduce dystocia, especially for heifers, minimizing commercial losses [10]. The pelvic measurements can successfully identify abnormally small or misshaped pelvises $[2,3]$. Two measures are fundamental for pelvimetry for pelvic classification: true 
conjugate (sacropubic) diameter and median transverse (bi-iliac) breadth. The occurrence of dystocia can be reduced by evaluating mainly the two measures since the fetus must be smaller than or equal to the pelvic dam capacity for a normal delivery $[9,10]$. There is limited usage of pelvimetry to predict calving difficulty. So, the prospect for analyses to evaluate the clinical efficiency of the pelvimeter to predict dystocia is highly needed [9].

Forensic age estimation from unidentified corpses, fossils, and skeletons for identification was an innovative feature of forensic science and paleontology. The significance of the bones is the keeping identifying features for an extended period, so the bony features are included in the indicators that reliably characterize the species, age, and sex belonging to the object of research [12]. The most informative thing to determine the species, time of death, and age is the skeleton because changes in the bones are stored for a very long time, while soft tissue is subjected to rotting [13]. To our knowledge, there is a sacristy of information on pelvimetry and parity in Egyptian buffaloes.

The present work aimed to throw the light deeply on the relationship between the pelvic measurements and the number of births of Egyptian buffaloes. This study will focus on pelvic measurements and how to use these measurements to estimate age and determine a prediction equation for the number of birth. The internal pelvimetry (Conjugate and transverse diameter) was used to determine pelvic area and its association with dystocia. The research question is, what are the most strongly correlated variables with the number of birth.

\section{METHODOLOGY}

The study was conducted on 36 buffaloes of native breed (nullipara, $n=14$ and pluripara, $n=22$ with 1-4 births aged between $<15$ months, $n=15$ and 65 months, $\mathrm{n}=21$ ). The animals were apparently healthy, and the management record and breeding history of the animals prior to slaughter were fully known. Immediately after slaughter, each carcass was eviscerated, and all soft tissues were removedadditional cleaning of the intact pelvis comprising the removal of genital organs, urinary bladder, and rectum. Skin and flesh were manually removed using dissecting tools. The internal pelvic measurements were obtained using the Rice pelvimeter (Lane Manufacturing Inc., Colorado, USA) described by Rice and Wiltbank [14].
The measuring tape (Tailor's tape) was used to determine the external pelvimetry.

\section{Ethical Statement}

All experiments have been conducted as per the Institutional Animal Ethics Committee guidelines, Faculty of Veterinary Medicine, Cairo University, Egypt.

\section{External Pelvimetry}

The distance between two ischiatic tuberosities and between sacral tubercles was measured. The distance between the acetabulum and coxal tuber and the dimensions (length and width) of obturator foramen were recorded. Length of pelvic symphysis was taken.

\section{Internal Pelvimetry}

The transverse breadth (pelvic width) was the horizontal distance between the shaft of the ilium at the widest part (psoas tubercles), and conjugate diameter (pelvic height) was measured as the distance from the cranial end of the pubic symphysis to the junction of the third and fourth sacral segments [15]. The pelvic area was calculated by multiplying the pelvic height with the width, which results in an ellipsoidal area which was calculated as such whereby: Ellipsoidal area $=($ width + height)/2 $\mathrm{x} \pi[14]$.

\section{Statistical Analysis}

All statistical analyses were performed with $\mathrm{SAS}^{\circledR}$ version 9.4 [16]. Statistical significance was set at $P$ $<0.05$, and all data were presented as the mean \pm standard error of the mean (SEM). To test correlations between the number of birth and each of the predictor variables, each variable was analyzed for normality through Shapiro-Wilk's test. When bivariate normality was verified, data were analyzed through a Pearson correlation (correlation coefficient $=r$ ). Results that did not follow this assumption were analyzed using a Spearman rank correlation (correlation coefficient $=\rho$ ). All multiple testing $p$-values for correlation analyses were adjusted to control the false discovery rate (FDR) using the Benjamini-Hochberg procedure [17].

A multiple Poisson regression model (with log-link) was developed using the PROC HPGENSELECT package in SAS with a backward elimination technique to select the set of predictors that could best estimate the number of births. In this model, the number of birth (followed Poisson distribution) was considered the response variable, while age (in months) and nine pelvimetry measurements were considered predictor variables. 


\begin{tabular}{|c|c|c|c|c|c|c|c|c|c|c|c|c|}
\hline \multirow{3}{*}{\multicolumn{2}{|c|}{ 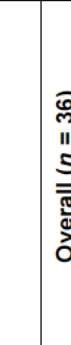 }} & 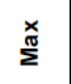 & $\begin{array}{l}8 \\
\text { ஸे }\end{array}$ & চ̊. & 怘 & $\begin{array}{l}\text { p్ } \\
\stackrel{d}{\sim}\end{array}$ & 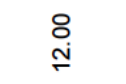 & 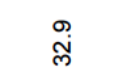 & $\begin{array}{l}\stackrel{8}{\text { N }} \\
\text { }\end{array}$ & 足 & 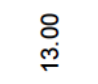 & $\stackrel{\circ}{\circ}$ \\
\hline & & $\stackrel{5}{\Sigma}$ & $\begin{array}{l}\stackrel{8}{\dot{m}} \\
\stackrel{9}{2}\end{array}$ & $\stackrel{\text { O్ }}{6}$ & $\begin{array}{l}\text { 吊 } \\
\text { ڤn }\end{array}$ & $\begin{array}{l}\stackrel{8}{8} \\
+\end{array}$ & $\stackrel{\leftrightarrow}{\circ}$ & $\begin{array}{l}\infty \\
\infty \\
\stackrel{\infty}{\sim}\end{array}$ & $\begin{array}{l}8 \\
\stackrel{8}{\circ}\end{array}$ & $\begin{array}{l}\text { ৪ } \\
\text { ஸे }\end{array}$ & $\underset{\infty}{\substack{\infty \\
\infty}}$ & $\underset{0}{\stackrel{8}{0}}$ \\
\hline & & 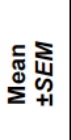 & 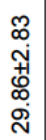 & 总 & $\begin{array}{l}\infty \\
0 \\
0 \\
01 \\
\infty \\
\infty \\
\infty\end{array}$ & $\begin{array}{l}\hat{m} \\
0 \\
01 \\
0 \\
0 \\
\stackrel{0}{\leftarrow}\end{array}$ & $\begin{array}{l}\stackrel{0}{0} \\
\text { O⿱艹 } \\
\stackrel{0}{0} \\
\end{array}$ & 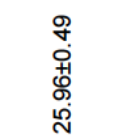 & 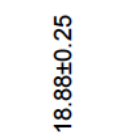 & 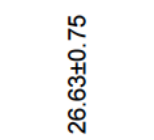 & $\begin{array}{l}\stackrel{n}{\circ} \\
\stackrel{0}{+1} \\
\stackrel{+}{\leftarrow}\end{array}$ & $\begin{array}{l}\stackrel{N}{0} \\
\stackrel{0}{+1} \\
\stackrel{N}{n}\end{array}$ \\
\hline \multirow{3}{*}{\multicolumn{2}{|c|}{$\begin{array}{l}0 \\
\text { II } \\
\text { a }\end{array}$}} & 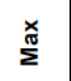 & 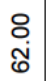 & 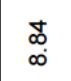 & 怘 & \& & $\stackrel{\stackrel{8}{ }}{=}$ & 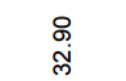 & 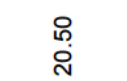 & $\frac{8}{\dot{m}}$ & 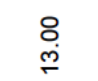 & $\underset{\substack{0 \\
\infty \\
\infty}}{ }$ \\
\hline & & $\frac{5}{\Sigma}$ & $\begin{array}{l}8 \\
\dot{\sim} \\
i\end{array}$ & 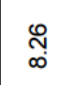 & 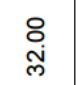 & $\begin{array}{l}\stackrel{\circ}{\circ} \\
\stackrel{\leftrightarrow}{\leftarrow}\end{array}$ & $\stackrel{R}{\stackrel{R}{r}}$ & 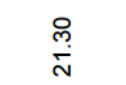 & 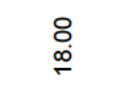 & 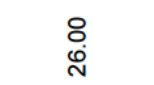 & 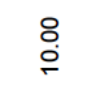 & $\underset{\sim}{\text { Oִ }}$ \\
\hline & & 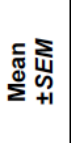 & $\begin{array}{l}0 \\
0 \\
0 \\
01 \\
0 \\
0 \\
0 \\
0\end{array}$ & 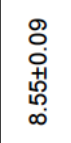 & $\begin{array}{l}\text { \& } \\
\text { Òं } \\
\stackrel{+}{N} \\
\text { స్ }\end{array}$ & 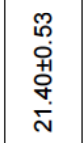 & $\begin{array}{l}\bar{n} \\
0 \\
0+1 \\
6 \\
\infty \\
\infty\end{array}$ & $\begin{array}{l}\widetilde{\infty} \\
\stackrel{+}{0} \\
\stackrel{\infty}{\infty} \\
\stackrel{N}{N}\end{array}$ & 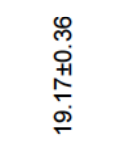 & $\begin{array}{l}\infty \\
\stackrel{\infty}{0} \\
0+1 \\
\infty \\
\infty \\
\infty \\
\sim\end{array}$ & 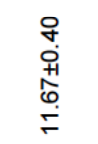 & 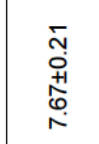 \\
\hline \multirow{3}{*}{\multicolumn{2}{|c|}{$\frac{5}{0}$}} & $\begin{array}{l}\sum_{\tilde{m}}^{\infty} \\
\Sigma\end{array}$ & $\begin{array}{l}8 \\
\dot{r} \\
\dot{f}\end{array}$ & ঃั & 号 & $\begin{array}{l}\text { p. } \\
\stackrel{\omega}{\text { N }}\end{array}$ & 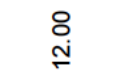 & $\frac{8}{\dot{m}}$ & $\begin{array}{l}\text { ৪ } \\
\text { ஸे }\end{array}$ & $\begin{array}{l}\text { ৪ } \\
\stackrel{\text { N }}{ }\end{array}$ & 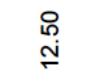 & $\stackrel{\circ}{\circ}$ \\
\hline & & $\stackrel{5}{\Sigma}$ & $\begin{array}{l}8 \\
\dot{y} \\
\text { D. }\end{array}$ & $\underset{\infty}{\infty}$ & 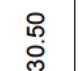 & $\begin{array}{l}\text { ৪ } \\
\text { ○् }\end{array}$ & 品 & 空 & 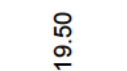 & 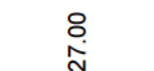 & 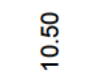 & 只 \\
\hline & & 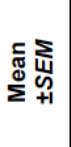 & 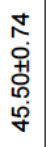 & 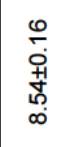 & 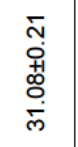 & $\begin{array}{l}\stackrel{0}{\circ} \\
\stackrel{i}{0} \\
\stackrel{0}{0} \\
\text { Ni }\end{array}$ & 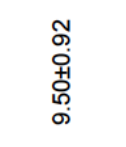 & 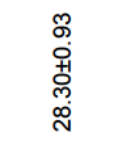 & 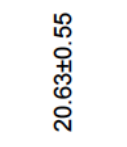 & 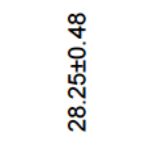 & $\begin{array}{l}\bar{m} \\
\text { o } \\
\text { pi } \\
\stackrel{5}{+}\end{array}$ & 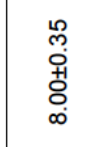 \\
\hline & \multirow{3}{*}{$\begin{array}{l}\text { O } \\
\text { II } \\
\text { s } \\
\text { N }\end{array}$} & 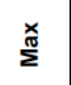 & $\begin{array}{l}\stackrel{8}{o} \\
\dot{p}\end{array}$ & $\begin{array}{l}\underset{\infty}{*} \\
\infty\end{array}$ & స్లి & $\begin{array}{l}\stackrel{\infty}{.0} \\
\stackrel{N}{N}\end{array}$ & $\begin{array}{l}\infty \\
\infty \\
\infty\end{array}$ & $\begin{array}{l}R \\
\substack{\infty \\
N}\end{array}$ & $\begin{array}{l}\text { : } \\
\text { مٌ }\end{array}$ & ষ্ণে & 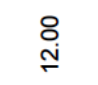 & $\stackrel{\circ}{\circ}$ \\
\hline & & $\frac{5}{\Sigma}$ & $\begin{array}{l}0 \\
\text { D. } \\
\text { p. }\end{array}$ & 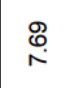 & $\begin{array}{l}\stackrel{8}{\infty} \\
\stackrel{\infty}{N}\end{array}$ & $\begin{array}{l}\text { ్ㅗ } \\
\text { }\end{array}$ & 品 & ه্. & $\begin{array}{l}\text { 只 } \\
\text { م. }\end{array}$ & : & $\begin{array}{l}\text { 융 } \\
\stackrel{0}{2}\end{array}$ & $\underset{\sim}{\stackrel{8}{r}}$ \\
\hline & & 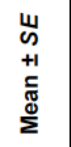 & 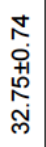 & $\begin{array}{l}\text { 웅 } \\
\text { ò } \\
\text { † } \\
\infty \\
\infty\end{array}$ & 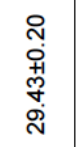 & $\begin{array}{l}0 \\
0 \\
\stackrel{+}{+1} \\
\stackrel{N}{N} \\
\stackrel{N}{N}\end{array}$ & $\begin{array}{l}0 \\
0 \\
0 \\
01 \\
\infty \\
\infty \\
\infty\end{array}$ & $\begin{array}{l}\stackrel{N}{N} \\
\text { ì } \\
0 \\
\infty \\
\infty\end{array}$ & 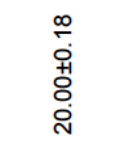 & $\begin{array}{l}\infty \\
m \\
\stackrel{\infty}{+} \\
\substack{\infty \\
\infty \\
\tilde{N} \\
\sim}\end{array}$ & $\begin{array}{l}\bar{N} \\
\text { ò } \\
\stackrel{p}{m} \\
\stackrel{\oplus}{=}\end{array}$ & 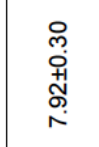 \\
\hline \multirow{3}{*}{\multicolumn{2}{|c|}{$\begin{array}{l}0 \\
\text { II } \\
\text { s } \\
\sigma\end{array}$}} & $\sum_{\substack{\sigma \\
\Sigma}}^{\frac{x}{2}}$ & $\begin{array}{l}\stackrel{8}{\dot{j}} \\
\text { and }\end{array}$ & $\stackrel{\leftrightarrow}{\stackrel{\leftrightarrow}{\circ}}$ & $\begin{array}{l}\text { o } \\
\stackrel{\infty}{\infty} \\
\text { N }\end{array}$ & $\frac{\circ}{\stackrel{N}{\dot{n}}}$ & $\underset{\infty}{\stackrel{N}{\infty}}$ & $\begin{array}{l}\stackrel{q}{d} \\
\stackrel{N}{n}\end{array}$ & $\begin{array}{l}\stackrel{\circ}{\circ} \\
\stackrel{0}{\circ}\end{array}$ & 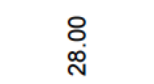 & 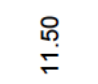 & $\underset{\infty}{\stackrel{0}{0}}$ \\
\hline & & $\underline{\Sigma}$ & $\begin{array}{l}8 \\
\stackrel{i}{ }\end{array}$ & 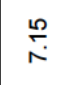 & $\begin{array}{l}\stackrel{8}{\infty} \\
\stackrel{\infty}{N}\end{array}$ & 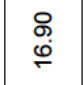 & $\underset{+}{\stackrel{\leftrightarrow}{*}}$ & $\begin{array}{l}\stackrel{\infty}{\infty} \\
\infty\end{array}$ & $\begin{array}{l}\stackrel{B}{\leftrightarrow} \\
\stackrel{n}{\sim}\end{array}$ & $\begin{array}{l}8 \\
\stackrel{\leftrightarrow}{\oplus}\end{array}$ & 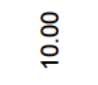 & 员 \\
\hline & & 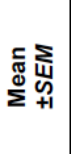 & 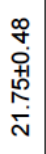 & 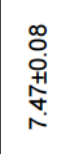 & 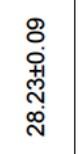 & $\begin{array}{l}0 \\
0 \\
0 \\
01 \\
0 \\
0 \\
\infty \\
\infty\end{array}$ & $\begin{array}{l}\hat{b} \\
0 \\
01 \\
0 \\
0 \\
0\end{array}$ & 旡 & 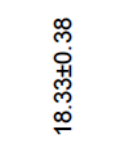 & $\begin{array}{l}\bar{n} \\
0 \\
+1 \\
\stackrel{N}{N} \\
\stackrel{N}{n}\end{array}$ & 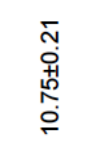 & 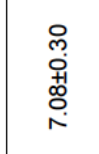 \\
\hline \multirow{3}{*}{\multicolumn{2}{|c|}{$\begin{array}{l}\text { t } \\
\text { II } \\
5 \\
0\end{array}$}} & 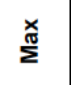 & 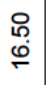 & : & 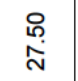 & $\frac{O}{i}$ & 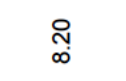 & $\begin{array}{l}\text { @ } \\
\stackrel{\leftrightarrow}{\sim}\end{array}$ & : & ষ্ণ & 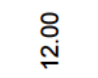 & 另 \\
\hline & & 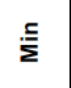 & 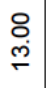 & ?ु. & 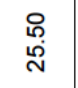 & $\begin{array}{l}\stackrel{8}{+} \\
\dot{+}\end{array}$ & 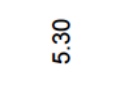 & ষ্ণ & ه. & $\begin{array}{l}\stackrel{8}{+} \\
\stackrel{\text { L }}{ }\end{array}$ & $\underset{\infty}{\stackrel{\infty}{\infty}}$ & $\underset{0}{8}$ \\
\hline & & 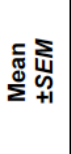 & 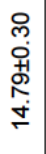 & 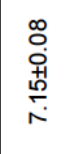 & 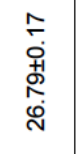 & 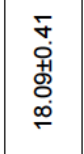 & 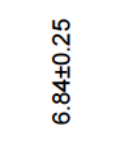 & 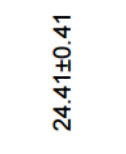 & 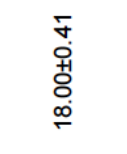 & 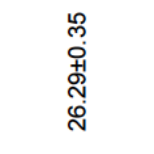 & 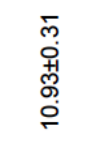 & 兽 \\
\hline \multicolumn{3}{|c|}{ 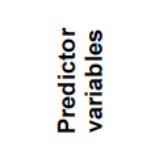 } & 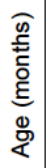 & 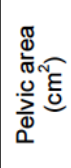 & 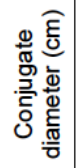 & 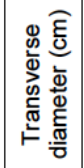 & 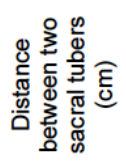 & 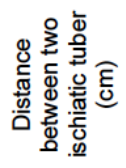 & 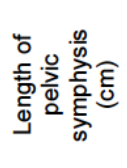 & 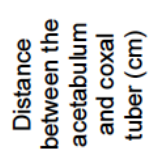 & 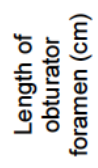 & 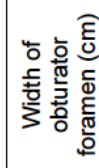 \\
\hline
\end{tabular}


Table 2: The Correlation Analysis Tests between the Number of Birth and each of the Predictor Variables ( $n=36$ ). Significant Results at $P<0.05$ if $\left({ }^{1}\right)$

\begin{tabular}{|c|c|c|}
\hline Predictor variables & Spearman rank $(\rho)^{1}$ & $\boldsymbol{p}$-value \\
\hline \hline Age (months) & 0.96 & $<0.0002$ \\
\hline Conjugate diameter $(\mathrm{cm})$ & 0.96 & $<0.0002$ \\
\hline Pelvic Area $\left(\mathrm{cm}^{2}\right)$ & 0.89 & $<0.0002$ \\
\hline Transverse diameter $(\mathrm{cm})$ & 0.74 & $<0.0002$ \\
\hline Distance between ischiatic tuberosities $(\mathrm{cm})$ & 0.64 & $<0.0002$ \\
\hline Distance between sacral tubercles $(\mathrm{cm})$ & 0.62 & $<0.0002$ \\
\hline Distance between acetabulum and coxal tuber $(\mathrm{cm})$ & 0.57 & 0.0004 \\
\hline Length of pelvic symphysis $(\mathrm{cm})$ & 0.50 & 0.0024 \\
\hline Width of obturator foramen $(\mathrm{cm})$ & 0.35 & 0.0427 \\
\hline Length of obturator foramen $(\mathrm{cm})$ & 0.19 & 0.2644 \\
\hline
\end{tabular}

${ }^{1}$ All $P$-values were adjusted to control the false discovery rate using the Benjamini-Hochberg procedure.

\section{RESULTS}

The age of animals used in this study ranged between 13 and 62 months. Descriptive statistics of the pelvic measurements are presented in Table 1 . The pelvic ellipsoidal area of the buffalo cows ranged between 6.43 and $9.00 \mathrm{~cm}^{2}$. The pelvic height was typically larger than pelvic width by about $40 \%$. The variable "number of births" was not following normal distribution (Shapiro-Wilk: $W=0.83, P<0.0001$ ). Therefore, Spearman rank correlation was used to test for correlation between the number of birth and each of the ten predictor variables (age and nine pelvimetry parameters). Results of correlation analysis tests are shown in Table 2.

\section{External Pelvimetry}

Strong positive linear relationships were found for the distance between ischiatic tuberosities and the distance between sacral tubercles with a correlation coefficient of 0.64 and 0.62 , respectively (Table 2 ). The width of obturator foramen had a weak relationship, while the length of obturator foramen had no significant correlation of 0.19 . The distance between the acetabulum and coxal tuber and the length of pelvic symphysis had a medium positive linear relationship with a correlation coefficient of 0.57 and 0.50 , respectively.

\section{Internal Pelvimetry}

The pelvic inlet in the Egyptian buffalo was oblique in the caudoventral direction. There was a significant correlation $(P<0.05)$ between the number of birth and all internal pelvic measurements (Table 2). The conjugate diameter and pelvic area had a strong positive linear relationship with a correlation coefficient of 0.96 and 0.89 , respectively, while the correlation coefficient for transverse diameter was 0.74 .

\section{Prediction of Number of Birth}

Multiple Poisson regression was performed - using a backward selection method - to ascertain the effects of age and nine pelvimetry measurements on the number of birth. The selected model was statistically significant $\left(\mathrm{X}_{(34)}^{2}=11.33, P<0.0001\right.$; adjusted $\mathrm{R}^{2}$ $=0.86$; Table 3 ) which consisted of intercept and conjugate diameter $(\mathrm{cm})$. The following linear combinations of predictor variables can predict the number of birth.

Natural log (number of birth $)=(0.4803 \times$ conjugate diameter in $\mathrm{cm}$ ) -13.97

Figure 1 provides the distribution of the predicted number of birth in each observed number of birth categories. Using the selected prediction model, the predicted number of birth with the associated $95 \%$ confidence limits for different conjugate diameters (ranged between 25.5 to $32.5 \mathrm{~cm}$ ) is presented in Figure 2.

\section{DISCUSSION}

The pelvic area is commonly calculated by multiplying the pelvic height with the width, which 
Table 3: Analysis of Parameter Estimates for Multiple Poisson Regression Tests using a Backward Elimination Technique to Select the Set of Predictors that Best Predict the Number of Birth. Selected Effects were Statistically Significant at $P<0.05$

\begin{tabular}{|c|c|c|c|c|c|c|c|}
\hline$\frac{\bar{\Phi}}{0}$ & Intercept & $\begin{array}{c}\text { Estimate } \\
-13.98\end{array}$ & $\begin{array}{c}S E \\
2.34\end{array}$ & -18.57 & -9.39 & $\begin{array}{c}\begin{array}{c}\text { Wald } \\
x_{(1)}^{2}\end{array} \\
35.61\end{array}$ & $\begin{array}{l}\boldsymbol{P} \text {-value } \\
<0.0001\end{array}$ \\
\hline $\bar{\infty}$ & Conjugate diameter $(\mathrm{cm})$ & 0.48 & 0.08 & 0.33 & 0.63 & 40.35 & $<0.0001$ \\
\hline \multirow{5}{*}{ 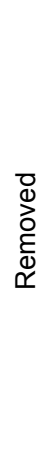 } & Age (months) & -0.02 & 0.07 & -0.16 & 0.11 & 0.13 & 0.7198 \\
\hline & Distance between two sacral tubers $(\mathrm{cm})$ & -0.11 & 0.18 & -0.45 & 0.24 & 0.37 & 0.5446 \\
\hline & Distance between two ischiatic tubers $(\mathrm{cm})$ & 0.00 & 0.07 & -0.13 & 0.13 & 0.00 & 0.9677 \\
\hline & Length of pelvic symphysis (cm) & 0.03 & 0.21 & -0.37 & 0.43 & 0.02 & 0.8831 \\
\hline & Distance between the acetabulum and coxal tuber $(\mathrm{cm})$ & -0.02 & 0.03 & -0.08 & 0.04 & 0.33 & 0.5672 \\
\hline
\end{tabular}

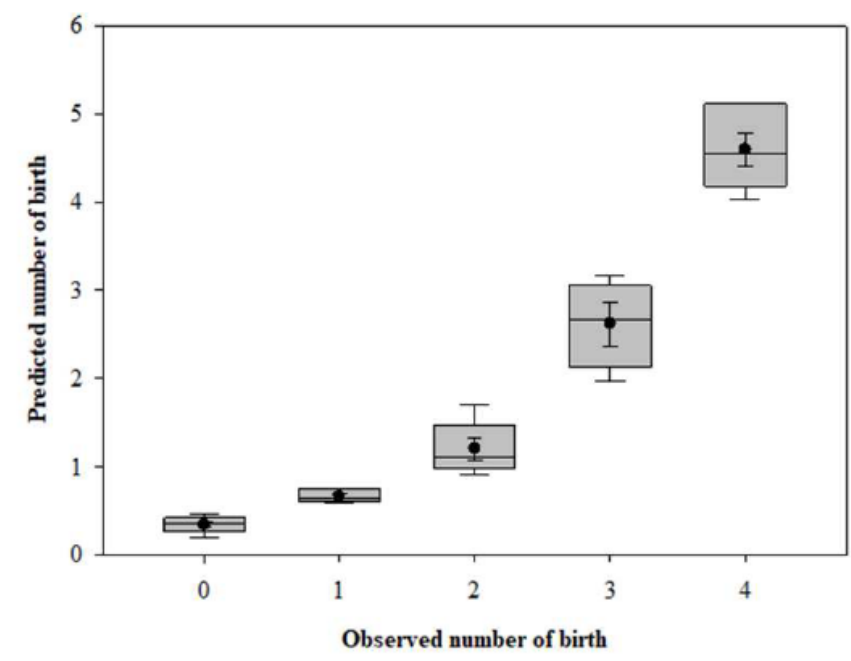

Figure 1: Box plot for the predicted number of birth in each category of the observed number of birth. Vertical lines refer to median while black dots refer to mean. Error bars around the dot refer to standard errors of the mean.

results in an ellipsoidal area $[14,18]$. That means the pelvic height is typically larger than the pelvic width. Similar results were found in Murrah, Jafarabadi, and Mediterranean breeds of buffaloes; these animals have a conjugate diameter larger than the transverse bi-iliac breadth, presenting pelvis with a similar anatomical form to an ellipse with a main vertical axis [19]. However, differences between the height of the pelvic inlet and the diagonal diameter of the pelvic inlet are not significant [20]. These different results may be due to the approaches used; pelvimetric dimensions achieved in carcasses and in vivo may lead to substantial differences. Although these differences are significant, it is very small for possible diversification of obstetric conduction during dystocia [21]. This signifies the reliability of the results obtained in animals when using the rice pelvimeter. The pelvis development level in heifers had a positive correlation with the advancing age of the buffalo heifers [22] and cattle [23]. In our study, the conjugate diameter increased progressively with the age and number of births. To our knowledge, this is the first study to outline the correlation between pelvimetry and the number of births in Egyptian buffaloes.

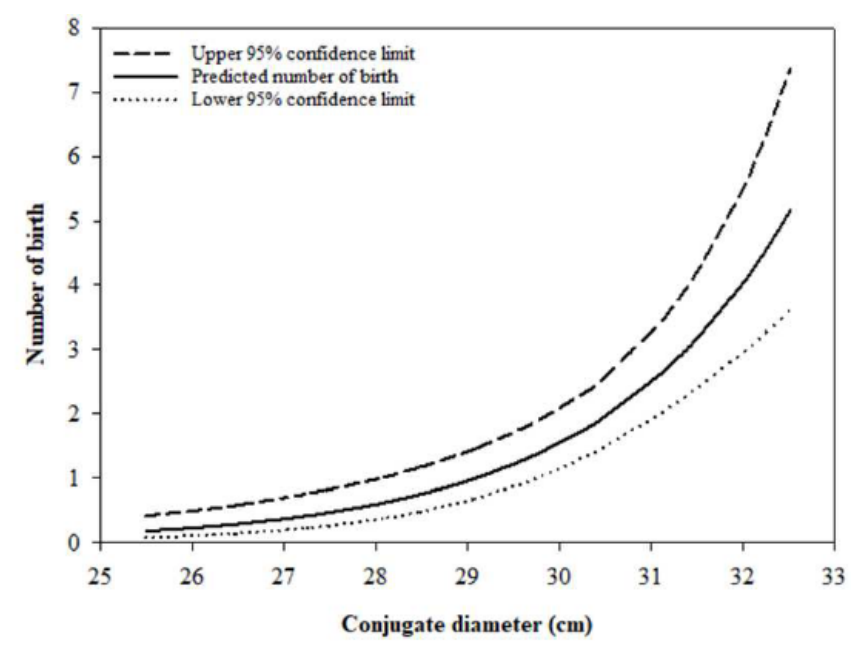

Figure 2: Predicted number of birth, upper and lower $95 \%$ confidence limits as estimated by Poisson regression model with the conjugate diameter $(\mathrm{cm})$ as a predictor variable. 
Pelvic area measurements can be divided by standard pelvic area/calf birth weight (PA/BWT) ratios to predict deliverable calf birth weight $[24,25]$. Recently, pelvic area/heifer weight (PA/WT) ratios were used as a test to select "easy calving" heifers, thereby increasing pelvic areas without increasing the size of the heifer [24]. Another way reported to be useful for predicting the dystocia of cattle at the time of calving is using a calving prediction equation that includes precalving maternal pelvic area and fetal hoof circumference [26]. The score calculated from this equation is designed to help producers decide which animals need assistance during calving.

\section{CONCLUSION}

In conclusion, the internal pelvic measurements positively correlate with the number of births and age in Egyptian buffaloes. Assessment of the pelvic area using the formula proposed for the internal pelvic area is important since it can confirm statistical differences in the areas of pelvic components between animals in different parities and ages. There is a very strong positive relationship between the conjugate diameter and the number of births. The larger the conjugate diameter, the higher the number of births. Thus, the age can be estimated depending on the conjugate diameter.

\section{CONFLICT OF INTEREST}

None of the authors have any conflict of interest to declare.

\section{DATA AVAILABILITY}

All data and materials and software applications or custom code are available from the authors on request.

\section{CODE AVAILABILITY}

The SAS code used to conduct the described analyses can be requested from Hisham $A$. Abdelrahman.

\section{ACKNOWLEDGEMENT}

The authors are grateful to the general scientific research department of Cairo University, Egypt, for support and assistance.

\section{REFERENCES}

[1] Jeengar K, Purohit GN, Mehta JS, Choudhary V, Nirwan LK. $A$ retrospective study on the incidence of dystocia in cattle and buffaloes at referral center. Theriogenology Insight 2015; 5(1): 41-45.

https://doi.org/10.5958/2277-3371.2015.00004.2

[2] Holm DE, Webb EC, Thompson PN. A new application of pelvis area data as culling tool to aid in the management of dystocia in heifers. J Anim Sci 2014; 92(5): 2296-2303.

https://doi.org/10.2527/jas.2013-6967

[3] Deutscher $\mathrm{GH}$. Reducing calving difficulty by heifer and sire selection and management. In Range Beef Cow Symposium. 1995; p. 183.

[4] Studdert VP, Gay CC, Hinchcliff KW. Saunders comprehensive veterinary dictionary e-book. Elsevier Health Sciences 2020.

[5] Silva RL, Oliveira WD, Biagiotti D, Ferreira GJ. Pelvimetry of multiparous Nellore cows in the cycling and early puerperal stages. Pes Vet Bras 2019; 39(5): 348-354. https://doi.org/10.1590/1678-5150-pvb-5466

[6] Waziri MA, Gabakan YJ, Mustapha AR. Pelvimetry of Kuri and Bunaji cows in Maiduguri metropolitan slaughterhouse, northern Nigeria. Sokoto J Vet Sci 2011; 9(2): 7-10.

\section{https://doi.org/10.4314/sokjvs.v10i2}

[7] Nogalski Z. Effect of selected factors on the course of parturition in Holstein-Friesian heifers. EJPAU 2002; 5(2). Doi: http://www.ejpau.media.pl/volume5/issue2/animal/art03.html

[8] Derivaux J, Ectors F. Pathophysiology of gestation and veterinary obstetrics. Maisons-Alfort: Éditions du Point Vétérinaire 1984; p. 273.

[9] Hiew MW, Constable PD. The usage of pelvimetry to predict dystocia in cattle. J Vet Malaysia 2015; 27(2): 1-4.

[10] Oliveira PC, Bombonato PP, Balieiro JC. Pelvimetry in females Nellore. Brazilian J Vet Res Anim Sci 2003; 40(4): 297-304.

https://doi.org/10.1590/S1413-95962003000400009

[11] Singh B. Dyce, Sack, Wensing's Textbook of veterinary anatomy. 5th ed. Pennsylvania: Saunders Elsevier 2016; p. 827.

[12] Monfared AL. Gross anatomical measurements of the head region of the Iranian native cattle (Bos taurus) and their clinical value for regional anesthesia. Global Veterinaria 2013; 10(2): 219-222.

[13] Yatsenk IV, Tkachuk SA, Busol LV, Bondarevsky MM, Zabarna IV, Biben IA. X-ray densitometric indices of proximal phalanx, medial phalanx and ungular pelvic limb bones as criteria for age diagnosis of cattle in forensic veterinary expertise. Regul Mech Biosyst 2019; 10(2): 197-202. https://doi.org/10.15421/021929

[14] Rice LE, Wiltbank JN. Factors affecting dystocia in beef heifers. J Am Vet Med Asso 1972; 161(11): 1348-1358.

[15] Colburn DJ, Deutscher GH, Nielsen MK, Adams DC. Effects of sire, dam traits, calf traits, and environment on dystocia and subsequent reproduction of two-year-old heifers. J Anim Sci 1997; 75(6): 1452-1460.

\section{https://doi.org/10.2527/1997.7561452x}

[16] Statistical Analysis Systems, SAS. The SAS System for Windows, Release 9.4. Statistical Analysis Systems Institute, Cary, NC, 2013; p. 556.

[17] Benjamini $Y$, Hochberg $Y$. Controlling the false discovery rate: A practical and powerful approach to multiple testing. $\mathrm{J}$ R Stat Soc Series B 1995; 57(1): 289-300. https://doi.org/10.1111/j.2517-6161.1995.tb02031.x

[18] Morrison DG, Williamson WD, Humes PE. Estimates of heritabilities and correlations of traits associated with pelvic area in beef cattle. J Anim Sci 1986; 63(2): 432-437. https://doi.org/10.2527/jas1986.632432x

[19] Rezende MD, Ferraz PC, Carneiro PS, Malhado CM. Phenotypic diversity in buffalo cows of the Jafarabadi, 
Murrah, and Mediterranean breeds. Pesq Agropec Bras 2017; 52(8): 663-669.

https://doi.org/10.1590/s0100-204x2017000800012

[20] Tsousis G, Heun C, Becker M., Bollwein H. Application of computed tomography for the evaluation of obstetrically relevant pelvic parameters in German Holstein-Friesian cows. Theriogenology 2010; 73(3): 309-315. https://doi.org/10.1016/j.theriogenology.2009.09.014

[21] Kolkman I, Hoflack G, Aerts S, Murray RD, Opsomer G, Lips D. Evaluation of the Rice pelvimeter for measuring pelvic area in double-muscled Belgian Blue cows. Livest Sci 2009; 121(2-3): 259-266.

https://doi.org/10.1016/j.livsci.2008.06.022

[22] Dhaliwal AS, Dugwekar YG, Sharma RD. Invivo pelvimetry in buffaloes (Bos Bubalus). Theriogenology 1981; 15(5): 501504

https://doi.org/10.1016/0093-691X(81)90093-5
[23] Brown JE, Brown CJ, Butts WT. A discussion of the genetic aspects of weight, mature weight, and rate of maturing in Hereford and Angus Cattle. J Anim Sci 1972; 34(4): 525-537. https://doi.org/10.2527/jas1972.344525x

[24] Basarab JA, Rutter LM, Day PA. The efficacy of predicting dystocia in yearling beef heifers: I. Using ratios of pelvic area to birth weight or pelvic area to heifer weight. $J$ Anim Sci 1993; 71(6): 1359-1371. https://doi.org/10.2527/1993.7161359x

[25] Johnson SK, Deutscher GH, Parkhurst A. Relationships of pelvic structure, body measurements, pelvic area, and calving difficulty. J Anim Sci 1988; 66(5): 1081-1088. https://doi.org/10.2527/jas1988.6651081x

[26] Ko JC, Ruble MV. Using maternal pelvis size and fetal hoof circumference to predict calving difficulty in beef cattle. Vet Med 1988; 85: 1030-1036.

https://doi.org/10.6000/1927-520X.2022.11.01

(C) 2022 Sary et al.; Licensee Lifescience Global.

This is an open access article licensed under the terms of the Creative Commons Attribution License (http://creativecommons.org/licenses/by/4.0/) which permits unrestricted use, distribution and reproduction in any medium, provided the work is properly cited. 